\title{
Detection in a four-observation two-channel task
}

\author{
EDWIN KAY and JOSEPH WEBER \\ Lehigh University, Bethlehem, Pennsylvania 18015
}

\begin{abstract}
Two models are proposed for a four-observation two-channel forced-choice paradigm of signal detection. The first model, an extension of signal detection theory, fails, while the second model, an extension of the classical decision threshold model, succeeds.
\end{abstract}

There have been several attempts to extend signal detection theory (SDT. Green \& Swets, 1966) to multiple observations by a single observer. One such study, by Swets and Green (1961), used the theory of sequential analysis to extend SD'T and applied the resulting theory to an experimental paradigm where the subject controls the number of observations preceding a decision. They found that uninstructed subjects did not integrate information from successive observations but could integrate information if given instructions to do so. In a similar study, Swets and Birdsall (1967) found that subjects who controlled the number of observations were able to use an optimal process of cumulating sensory information over successive observations, although a consistent decision bias was displayed. Doherty and Keeley (1969) investigated multiple observations in a visual recognition task and were successful in making predictions of four-look performance from one-look data. Kinchla (1969) also used a detection model to imvestigate multiple observation tasks with auditory stimuli. He suggests that simultaneous multiple observations are possible whereby "channel uncertainty" in such a task can then be equated to "temporal uncertainty" in a sequential multipleobservation task.

Swets, Shipley, McKey, and Green (1959) present two models for multiple observations; one model is an extension of SD' assuming integration of information, while the other model is an extension of the classical decision-threshold model. They found that a subject's accuracy increases as the number of observations preceding a decision increases, a prediction made by both models. Although the two models differ in the quantitative details of the prediction of increased accuracy with increased observations, this difference is not easily tested in the paradigm used.

In the present study, we develop a new paradigm for multiple observations. We also derive extensions

Requests for reprints should be sent to Edwin Kay, Department of Psychology, Lehigh University, Bethlehem. Pennsylvania 18015. The work of the senior author was supported in part by National Institutes of Health Postdoctoral Research Fellowship 1-F02GM43917-01. of the SD' and threshold models that apply to the new paradign.

\section{METHOD}

\section{Procedure and Apparatus}

The sound-proofed experimental room had a comfortable chair fatcing a wall mounted with two $24-\mathrm{V}$ lights. $1 \mathrm{in}$. in diam, one green and one red. BRS Foringer digital logic equipment was used to present, for $7 \mathrm{sec}$, either the red or the green light. Simultaneously, white noise with a $1.3-\mathrm{kHz}$ tone present or absent was presented over earphones.

A test session consisted of 600 trials (without feedback) of the following four-observation two-channel forced-choice procedure (FOFC):

Each trial consisted of four observations of a simultaneous visual and auditory display. Each observation lasted $.7 \mathrm{sec}$, with $.7 \mathrm{sec}$ between observations. These sets of four observations are of two types: (a) the red light occurs with every observation of signal and the green light occurs with every observation of noise, and (b) the green light occurs with every observation of signal and the red light oceurs with every observation of noise. There are $n$ observations of signal and $4 n$ observations of noise with $n=1,2$, or 3 .

The red and green lights represent two channels of information. On each trial. the choice of the channel with signal (i.e., whether the observations are of Type a or Type b) is random, with $p=.5$. The observer's task on a trial is to determine which channel had the signal, i.e., whether on that trial green was associated with signal (and red with noise) or red with signal (and green with noise).

Using $S$ to denote signal and $N$ to denote noise, we denote the various stimulus sequences comprising a trial by SNNN. SNSN, etc.

There were seven test sessions. The 600 observations of each test session consisted of a random order of 150 observations each of four stimulus sequences. The sequences used in each test session are shown in lable 1. Before and after each test session. 100 two-alternative forced-choice (2AFC) trials were presented. The lirst set of $1002 \mathrm{AFC}$ trials was preceded by a cue tone which gave the observers an opportunity to hear the signal before the trial began. Gundy $(1961)$ found that this procedure leads to a stable pertormance. Each 2AFC trial was initiated by the observer and consisted of an interval signaled by the green light followed $.7 \mathrm{sec}$

Table 1

Stimulus Sequences for Each Test Session

\begin{tabular}{cc}
\hline Session & \multicolumn{1}{c}{ Stimuli } \\
\hline 1 & SSSN, NNNS, NSSS, SNNN \\
2 & SSNN, NNSS, SNSN, NSNS \\
3 & SSNS, NNSN, SNSS, NSNN \\
4 & SSSN, NNNS, SSNN, NNSS \\
5 & SSNN, NNSS, SNNS, NSSN \\
6 & NSSS, SNNN, SNNS, NSSN \\
7 & SNSS, NSNN, SNNS, NSSN \\
\hline
\end{tabular}


Table 2

The Number of Correct Decisions (Out of 150) by Each Observer for Each Sequence of Each Test Session

\begin{tabular}{|c|c|c|c|}
\hline & \multirow{2}{*}{$\begin{array}{l}\text { Stimulus } \\
\text { Sequence }\end{array}$} & \multicolumn{2}{|c|}{ Subject } \\
\hline & & B.W. & J.W. \\
\hline Session 1 & $\begin{array}{l}\text { SSSN } \\
\text { NSSS } \\
\text { NNNS } \\
\text { SNNN }\end{array}$ & $\begin{array}{r}95 \\
112 \\
76 \\
55\end{array}$ & $\begin{array}{l}134 \\
128 \\
111 \\
106\end{array}$ \\
\hline Session 2 & $\begin{array}{l}\text { SSNN } \\
\text { NNSS } \\
\text { SNSN } \\
\text { NSNS }\end{array}$ & $\begin{array}{l}93 \\
83 \\
94 \\
86\end{array}$ & $\begin{array}{l}95 \\
95 \\
94 \\
96\end{array}$ \\
\hline Session 3 & $\begin{array}{l}\text { SSNS } \\
\text { SNSS } \\
\text { NNSN } \\
\text { NSNN }\end{array}$ & $\begin{array}{r}115 \\
111 \\
99 \\
108\end{array}$ & $\begin{array}{l}122 \\
120 \\
102 \\
115\end{array}$ \\
\hline Session 4 & $\begin{array}{l}\text { SSSN } \\
\text { SSNN } \\
\text { NNSS } \\
\text { NNNS }\end{array}$ & $\begin{array}{r}103 \\
83 \\
85 \\
78\end{array}$ & $\begin{array}{l}128 \\
126 \\
131 \\
111\end{array}$ \\
\hline Session 5 & $\begin{array}{l}\text { SSNN } \\
\text { SNNS } \\
\text { NNSS } \\
\text { NSSN }\end{array}$ & $\begin{array}{l}89 \\
91 \\
93 \\
86\end{array}$ & $\begin{array}{l}107 \\
108 \\
118 \\
119\end{array}$ \\
\hline Session 6 & $\begin{array}{l}\text { NSSS } \\
\text { SNNS } \\
\text { NSSN } \\
\text { SNNN }\end{array}$ & $\begin{array}{r}117 \\
98 \\
103 \\
89\end{array}$ & $\begin{array}{r}132 \\
121 \\
119 \\
99\end{array}$ \\
\hline Session 7 & $\begin{array}{l}\text { SNSS } \\
\text { SNNS } \\
\text { NSSN } \\
\text { NSNN }\end{array}$ & $\begin{array}{r}120 \\
103 \\
102 \\
82\end{array}$ & $\begin{array}{l}135 \\
115 \\
126 \\
102\end{array}$ \\
\hline
\end{tabular}

later by an interval signaled by the red light. The trial was terminated by the observer's pressing either the red or the green response key. The signal was assigned randomly to each light with equal probability.

Depending upon the model, one may make differential predictions for the probability of correctly choosing the channel with tone $[\mathrm{P}(\mathrm{C})]$ as a function of the stimulus sequence within a test session. We now propose two models for the FOFC paradigm and derive predictions that follow from each model. The first model is an extension of SDT, while the second model is an extension of the classical decision-threshold model.

\section{Model I: An Extension of SDT}

SDT assumes that a single observation is summarized by a real number which is a random variable having one of two possible distributions, depending on whether the observation is of signal or of noise. It is assumed that the signal and noise distributions are both normal. with variance $\sigma^{2}$ and with mean $\mu \mathrm{S}$ and $\mu \mathrm{N}$, respectively.

It is assumed that the subject acts like the "ideal observer" of statintical decision theory and adopts a decision rule that maximizes $P(C)$. It can be shown that, for any of the stimulus sequences of Table 1. the maximal value of $P(C)$ is $P\left[Z \geqslant\left(\mu_{S}-\mu_{N}\right) / \sigma\right]$, where $Z$ is a normal randon variable with zero mean and unit variance. (See Appendix A for a derivation.) Thus. Model I predicts the same value of $P(C)$ for all the stinulus sequences of Table 1 .

\section{Model II: An Extension of the Threshold Model}

Model II. which seems most compatible with the decision-threshold model but is also compatible with SDT, assumes that a channel will be chosen as having signal if any observation of that channel keads to a detection. If no detection is made, the observer guesses. If $p$ is the probability of detection on a trial and $n$ ohservations of the channel with signal are made, then the probability of being correct is

$$
\begin{aligned}
P(C) & =1-(1-p)^{n}+1 / 2(1-p)^{n} \\
& =1-1 / 2(1-p)^{n} .
\end{aligned}
$$

Thus Model II predicts that the probability of being correct on a trial will increase as a function of the number of observations of the channel with signal. This prediction is symbolized as follows: [SNNN, NSNN, NNSN, NNNS] < [SSNN, SNSN, SNNS, NSNS, NSSN, NNSS] < [NSSS, SNSS, SSNS, SSSN], where stimuli sequences within brackets (fl) are predicted to give the same value of $P(C)$ and $<$ is read as "gives a smaller value of $P(C)$ than."

It woulal have been desirable to present all 14 sequences in one test session in order to test the two models. However, a sufticient number of observations per sequence would require an inordinately long test session. Thus seven test sessions were run with 150 observations each of four trial sequences per session. With the exception of Sessions 2 and 5 , the two models make different predictions for the stimuli sequences used in a session.

The junior author and his wife served as observers. Training consisted of approximately $25 \mathrm{l} \cdot \mathrm{h}$ sessions of practice using a $2 \mathrm{AFC}$ procedure. Training terminated when a criterion of tive consecutive sessions with a stable detection rate of approximately .75 was obtained.

\section{RESULTS AND DISCUSSION}

For each observer, chi-square tests were used to determine if the probabilities of being correct before and after each test session differed. None of the 14 chi squares was signiticant at the .05 level. This was taken as evidence that the detection level was stable for each observer during each test session.

The number of correct decisions (out of 150) made by each observer for each of the four sequences used in each session are shown in Table 2. Chi-square tests were used to test the predictions of each model for each subject for each session. For example, in Session 1, Model II predicts [NNNS, SNNN] < [SSSN, NSSS]. Three chi-square tests were used to test this prediction. First, the prediction [NNNS, SNNN] was tested by performing a chi-square test on the 2 by 2 frequency table whose columns were labeled NNNS and SNNN, respectively, and whose rows were labeled correct and incorrect, respectively. The prediction [SSSN, NSSS] was tested in the same fashion. Finally, the frequency data for SSSN and NSSS were combined, as were the frequency data for NNNS and SNNN. A chi-square test was performed on the resulting 2 by 2 frequency table.

The results of the chi-square tests are shown in Table 3, and we can now consider the results of these chi-squared tests for each model.

The predictions of Model I fare very poorly in Sessions $1,4,6$, and 7 , and are in agreement with data from Sessions 2 and 5 . The data from Session 3 are equivocal with respect to Model I. In reviewing the datat for Model II, it must be kept in mind that 
Table 3

Values of Chi Square for Predictions of Each Model for Each Session

\begin{tabular}{|c|c|c|c|c|}
\hline \multirow[b]{2}{*}{ Session } & \multirow[b]{2}{*}{ Model(s) } & \multirow[b]{2}{*}{ Prediction } & \multicolumn{2}{|c|}{ Chi Square } \\
\hline & & & B.W. & J.W. \\
\hline \multirow[t]{4}{*}{1} & I & [SSSN, NNNS, SNNN, NSSS] & $49.03 * *$ & $22.23 * *$ \\
\hline & II & [SSSN, NSSS] & $4.50^{*}$ & 1.81 \\
\hline & & [NNNS, SNNN] & $5.96 *$ & .42 \\
\hline & & {$[$ NNNS, SNNN] $<$ [SSSN, NSSS $]$} & $39.13 * *$ & $20.96 * *$ \\
\hline 2 & I, II & [SSNN, NNSS, SNSN, NSNS] & 2.38 & .06 \\
\hline \multirow[t]{4}{*}{3} & I & [SSNS, SNSS, NNSN, NSNN] & 4.61 & $9.00 * *$ \\
\hline & II & [SSNS, SNSS] & .29 & .37 \\
\hline & & [NNSN, NSNN] & 1.26 & 2.81 \\
\hline & & {$[$ NNSN, NSNN $]<[$ SSNS, SNSS $]$} & 2.99 & $5.79^{*}$ \\
\hline \multirow[t]{3}{*}{4} & I & [SSSN, SSNN, NNSS, NNNS] & $9.78 * *$ & $11.07 * *$ \\
\hline & II & [SSNN, NNSS ] & .05 & .68 \\
\hline & & $[$ NNNS $]<$ [SSNN, NNSS $]<[$ SSSN $]$ & $9.38 * *$ & $10.49 * *$ \\
\hline 5 & I, II & [SSNN, SNNS, NNSS, NSSN] & .94 & 4.38 \\
\hline \multirow[t]{3}{*}{6} & I & [NSSS, SNNS, NSSN, SNNN] & $12.59 * *$ & $22.52 * *$ \\
\hline & II & [SNNS, NSSN] & .38 & .08 \\
\hline & & {$[$ SNNN] $<$ [SNNS, NSSN] $<$ [NSSS $]$} & $12.34 * *$ & $21.64 * *$ \\
\hline \multirow[t]{3}{*}{7} & 1 & [SNSS, NSNN, SNNS, NSSN] & $22.21 * *$ & $25.06 * *$ \\
\hline & II & [NSSN, SNNS] & .01 & 2.55 \\
\hline & & $[$ NSNN] $<$ [SNNS, NSSN $]<$ [SNSS $]$ & $22.05 * *$ & $22.39 * *$ \\
\hline
\end{tabular}

predictions of the form $[A, B]$ are corroborated by nonsigniticant chi-square tests, while predictions of the form $[\mathrm{A}]<[\mathrm{B}]$ are corroborated by significant chi-squared tests. The data from Sessions 2 and 4-7 are in perfect agreement with the prediction of Model II. In Session 1 for B.W., there are two signilicant chi-squared tests that are not predicted by Model II, and in Session 3 for B.W., there is a chi-squalred test that fails to be significant when it is predicted to be signiticant by Model II. Considering the number of chi-square tests carried out, the former failure could be attributed to Type I error. The latter failure could be attributed to Type II error. Moreover, in both Sessions 1 and 3, despite the aforementioned inconsistency. Model $1 \mathrm{I}$ is far more successfill than Model I.

To sum up, Model I fails except when its predictions are coincident with those of Model II. while Model II does quite well in predicting an increase in $P(C)$ as the number of observations of signal increases. Thus, Model I can be rejected in favor of Model II.

Model II is now considered in somewhat more detail. Model II predicts that if a sequence with $\mathbf{n}$ presentations of signal is observed, then

$$
P(C)=1-1 / 2(1-p)^{n},
$$

where $\mathrm{p}$ is the probability of detection. This equation may be rewritten as $\log \{2[1-P(C)]\}^{-1}=$ $n \log (1-p)^{-1}$, the equation of a straight line with zero intercept and slope $\log 1 /(1-p)$. Least-square fits of the above straight line were obtained for the data from each observer for each of the sessions that included sequences with 1,2 , and 3 presentations of signal (Sessions 4, 5, and 7), e.g., Session 1 was excluded since it did not have a sequence with two presentations of signal. The best-fit equations were then used to compute predicted values of $P(C)$. The predicted and obtained values of $P(C)$ are shown in Table 4 . In general, the predictions are quite close to the obtained data. This gives further credence to Model II.

The extension of SDT considered fails to account for the data in the present experiment. Thus the assumptions that are used to extend SDT are called into question. The most vulnerable assumption is that the observer has perfect memory for the information obtained from the four observations. Other than

Table 4

Predicted Values of $P(C)$ for Each Observer in Sessions 4, 6, and 7

\begin{tabular}{|c|c|c|c|c|c|c|}
\hline \multirow[b]{2}{*}{ Session } & \multirow[b]{2}{*}{$\mathrm{n}$} & \multicolumn{2}{|c|}{$\begin{array}{c}\text { Observer B.W. } \\
\text { P(C) }\end{array}$} & \multirow[b]{2}{*}{$\mathrm{n}$} & \multicolumn{2}{|c|}{$\begin{array}{c}\text { Observer J.W. } \\
\text { P(C) }\end{array}$} \\
\hline & & $\begin{array}{l}\text { Predic- } \\
\text { ted }\end{array}$ & Obtained & & $\begin{array}{l}\text { Predic- } \\
\text { ted }\end{array}$ & Obtained \\
\hline 4 & $\begin{array}{l}1 \\
2 \\
3\end{array}$ & $\begin{array}{l}.56 \\
.62 \\
.65\end{array}$ & $\begin{array}{l}.52 \\
.56 \\
.68\end{array}$ & $\begin{array}{l}1 \\
2 \\
3\end{array}$ & $\begin{array}{l}.69 \\
.81 \\
.88\end{array}$ & $\begin{array}{l}.74 \\
.86 \\
.85\end{array}$ \\
\hline 6 & $\begin{array}{l}1 \\
2 \\
3\end{array}$ & $\begin{array}{l}.61 \\
.70 \\
.76\end{array}$ & $\begin{array}{l}.59 \\
.67 \\
.78\end{array}$ & $\begin{array}{l}1 \\
2 \\
3\end{array}$ & $\begin{array}{l}.68 \\
.80 \\
.88\end{array}$ & $\begin{array}{l}.66 \\
.80 \\
.88\end{array}$ \\
\hline 7 & $\begin{array}{l}1 \\
2 \\
3\end{array}$ & $\begin{array}{l}.62 \\
.71 \\
.78\end{array}$ & $\begin{array}{l}.55 \\
.68 \\
.80\end{array}$ & $\begin{array}{l}1 \\
2 \\
3\end{array}$ & $\begin{array}{l}.70 \\
.82 \\
.89\end{array}$ & $\begin{array}{l}.68 \\
.80 \\
.90\end{array}$ \\
\hline
\end{tabular}

Note-Predictions were made by least-square fit of obtained values of $P(C)$ to the equation $\log 1 /[1-P(C)]=n \log 1 /(1-p)$. 
assuming a threshold model, which can be viewed as a no-memory SDT model, it is not clear how to modify this assumption in order to obtain a model that would account for the data.

\section{APPENDIX A}

Our SDT model predicts that for all the stimulus sequences used, $P\left[Z \geqslant\left(\mu_{S}-\mu_{N}\right), \sigma\right]$, where $Z$ is normal with zero mean and unit variance. To illustrate how this prediction is obtained, we derive this prediction in detail for a particular stimulus sequence. The derivations for other sequences are similar and lead to the same prediction, although some of the details may differ.

In the present experiment. consider, for example, a trial consisting of the visual presentation of three green lights followed by a red light simultaneous with the auditory information from the four observattions. This auditory information can be summarized by the real numbers $x_{1}, x_{2}, x_{3}$, and $x_{4}$. The subject must decide whether the stimulus sequence for the trial was SSSN or NNNS. Noting that SSSN and NNNS are equally likely on a trial. $P(C)$ may be maximized by choosing SSSN if and only if the likelihood ratio

$\ell_{S S S N}$ NNNS $\left(x_{1}, x_{2}, x_{3}, x_{4}\right)$

$$
=f^{\prime}\left(x_{1}, x_{2}, x_{3}, x_{4} \mid S S S N\right) / f\left(x_{1}, x_{2}, x_{3}, x_{4} \mid N N N S\right) \geqslant 1,
$$

where $f\left(x_{1}, x_{2}, x_{3}, x_{4} \mid S S S N\right)$ and $f\left(x_{1}, x_{2}, x_{3}, x_{4} \mid N N N S\right)$ are the joint distributions of $\left(x_{1}, x_{2}, x_{3}, x_{4}\right)$ given SSSN and NNNS, respectively. Now, assume the observations are independent. Then

$$
f\left(x_{1}, x_{2}, x_{3}, x_{4} \mid S S S N\right)=f\left(x_{1} \mid S\right) f\left(x_{2} \mid S\right) f\left(x_{3} \mid S\right) f\left(x_{4} \mid N\right)
$$

and

$$
f\left(x_{1}, x_{2}, x_{3}, x_{4} \mid N N N S\right)=f\left(x_{1} \mid N\right) f\left(x_{2} \mid N\right) f\left(x_{3} \mid N\right) f\left(x_{4} \mid S\right) .
$$

Thus

$\ell_{S S S N, \text { NNNS }}\left(x_{1}, x_{2}, x_{3}, x_{4}\right)=\ell_{S, N}\left(x_{1}\right) \ell_{S, N}\left(x_{2}\right) \ell_{S, N}\left(x_{3}\right) \ell_{N, S}\left(x_{4}\right)$. where

$$
\begin{aligned}
\boldsymbol{l}_{S, N}\left(x_{i}\right) & =\frac{f\left(x_{i} \mid S\right)}{f\left(x_{i} \mid N\right)}=\frac{\operatorname{Exp}\left[-\left(x_{i}-\mu S\right)^{2} / 2 \sigma^{2}\right]}{\operatorname{Exp}\left[-\left(x_{i}-\mu_{N}\right)^{2} / 2 \sigma^{2}\right]} \\
& \left.=\operatorname{Exp}\left[\mu_{S}-\mu_{N}\right)\left(x_{i}-1 / 2\left(\mu_{S}+\mu_{N}\right)\right) / \sigma^{2}\right]
\end{aligned}
$$

and

$$
\begin{aligned}
\boldsymbol{\ell}_{\mathrm{N} . S}\left(\mathrm{x}_{\mathrm{i}}\right) & =\mathrm{f}\left(\mathrm{x}_{\mathrm{i}} \mid \mathrm{N}\right) / \mathrm{f}\left(\mathrm{x}_{\mathrm{i}} \mid \mathrm{S}\right) \\
& \left.=\operatorname{Exp}\left[-\mu_{S} \mu_{\mathrm{N}}\right)\left(\mathrm{x}_{\mathrm{i}}-1 / 2\left(\mu_{\mathrm{S}}+\mu_{\mathrm{N}}\right)\right) / \sigma^{2}\right]
\end{aligned}
$$

thus

$\boldsymbol{\ell}_{\text {SSSN, NNNS }}\left(x_{1}, x_{2}, x_{3}, x_{4}\right)$

$$
\begin{aligned}
& \left.\qquad \operatorname{Exp}\left(\mu_{S}-\mu_{N}\right)\left(x_{1}+x_{2}+x_{3}-x_{4}-\mu_{S}-\mu_{N}\right)\right] \geqslant 0 \\
& \text { if and only if } x_{1}+x_{2}+x_{3}-x_{4} \geqslant \mu S+\mu N .
\end{aligned}
$$

Thus $P(C)$ may be maximized by deciding SSSN if and only if $x_{1}+$ $x_{2}+x_{3}-x_{4} \geqslant \mu_{S}-\mu_{N}$. In the situation where SSSN is presented, this maxinsum valuc of $P(C)$ is given by

$$
\begin{aligned}
P(C) & =P\left(x_{1}+x_{2}+x_{3} \cdot x_{4} \geqslant \mu S+\mu N \mid S S S N\right) \\
& =P(I \geqslant 0)
\end{aligned}
$$

where $T=x_{1}+x_{2}+x_{3}-x_{4}-\mu S-\mu N$, with $x_{1}, x_{2}, x_{3}$, and $x_{4}$ normal, $E\left(x_{1}\right)=E\left(x_{2}\right)=E\left(x_{3}\right)=\mu S . E\left(x_{4}\right)=\mu N$ and $\operatorname{Var}\left(x_{1}\right)=\sigma^{2}$ for $i=1,2,3,4$. The $T$ is normal. with $E(T)=2\left(\mu_{S}-\mu_{N}\right)$ and $\operatorname{Var}(T)=2 \sigma^{2}$. Then $Z=[T-E(T)] / \sqrt{\operatorname{Var}(T)}$ is normal with zero mean and unit variance.

$$
\mathrm{P}(\mathrm{C})=\mathrm{P}(\mathrm{I} \geqslant 0)=\mathrm{P}(\mathrm{Z} \geqslant(\mu \mathrm{S} \mu \mathrm{N}) / 0) .
$$

The ealculations for any stimulus sequence with three observations from one channel and one observation from the other channel are identical to those in the above example. The resulting decision rule is to say that the channel observed three times has signal if and only if $x_{1}+x_{2}+x_{3}-x_{4} \geqslant \mu S+\mu N$, where $x_{1}, x_{2}, x_{3}$ is the information from the channel observed three times and $x_{4}$ the intormation from the channel observed once. When a stimulus sequence consists of two observations of each channel, say $x_{1}$ and $x_{2}$ from one channel and $x_{3}$ and $x_{4}$ from the other channel, the decision rule would be to say the first channel has signal if and only if $x_{1}+x_{2} \geqslant x_{3}+x_{4}$. As noted above, this decision rule leads to the same value of $\mathrm{P}(\mathrm{C})$.

\section{REFERENCES}

Doherty, M. E.. \& KeEley. S. M. A Bayesian prediction of four-look recognition performance from one-look data. Perception \& Psychophysics, 1969, 5. 362-364.

Green, D. M., \& Swets, J. A. Signal detection theory and psychophysics. New York: Wiley, 1966.

Gundy, R. F. Auditory detection of an unspecitied signal. Journal of the Acoustical Society of America, 1961, 33, 1000-1012.

KinChla, R. A. Temporal and channel uncertainty in detection: A multiple observation analysis. Perception \& Psychophysics. $1969,5.129-136$.

Swets. J. A., \& Birdsall, T. G. Deferred decision in human signal detection: A preliminary experiment. Perception \& Psychophysics, 1967, 2, 15-28.

Swets, J. A.. \& Green, D. M. Sequential observations by human observers of signal in noise. In E. Cherry (Ed.). Information theon. London: Butterworths. 1961.

Swets, J. A.. Shipley, E. F., McKey, M. J., \& Green, D. M. Multiple observations of signal in noise. Journal of the Acoustical Society of America. 1959, 31, 514-521.

(Received for publication August 26, 1974; revision accepted December 4, 1974.) 